\title{
Dor e Fixadores Externos: Avaliação e Tratamento
}

\author{
Marta Imamura \\ Walter H. C. Targa \\ Manoel J. Teixeira \\ Lin Tchia Yeng \\ Satiko T. Imamura
}

\section{RESUMO}

A dor é a principal causa de retardo na aquisição funcional nos doentes em uso de fixadores externos. O objetivo do presente trabalho é o de avaliar os achados clínicos e os resultados do tratamento da dor em 20 doentes portadores de dor com características neuropáticas durante a vigência do uso de fixador externo tipo Ilizarov nos membros inferiores. O tratamento consiste na utilização de medicamentos analgésicos de ação central associados a anti-inflamatórios não hormonais e medidas de medicina física que incluem compressas de gelo, termoterapia profunda com ultrassom e neuroestimulação do sistema nervoso periférico. Um doente recebeu bloqueio simpático. Os resultados demonstraram alívio sintomático da dor neuropática em $80,9 \%$ dos casos, o que possibilitou a permanência do fixador nos membros e abordagem reabilitacional intensiva objetivando ganho funcional durante a vigência do tratamento ortopédico.

\section{UNITERMOS}

Dor. Fixadores Externos. Analgesia.

\section{SUMMARY}

Pain is the most important cause of the delay in the gain of functional state in patients using external fixators. The aim of this study is to evaluate the clinical findings and the results of pain treatment in 20 patients, presenting pain with neuropathic characteristics during the use of Ilizarov's external fixators in the lower limbs. All patients with deaferentiation symptoms were treated with central acting analgesics, antiinflamatory drugs and physical medicine modalities including ice packs, deep heat with ultrasound and neurostimulation of the peripheral nervous system. One patient recieved sympathetic block. Results demonstrated symptomatic relief of neuropathic pain in $80,9 \%$ of patients which permitted the continuation of the use of the external fixator, and an agressive rehabilitation approach aiming to increase of function during the orthopaedic treatment.

\section{KEY WORDS}

Pain. External Fixators. Analgesia.

\section{Introdução}

A fixação externa constitui um método de imobilização que se utiliza de fios percutâneos posicionados no tecido ósseo e ligados a conectores externos. O avanço tecnológico permitiu o incremento do material, da estabilidade e das técnicas operatórias, constituindo hoje um valioso instrumento no tratamento de fraturas e de diversas patologias ósteo-articulares. Os fixadores circulares do tipo Ilizarov anulam todas as forças que tendem a desviar os fragmentos ósseos, sendo extremamente estáveis.

As propriedades biomecânicas destes fixadores permitem a carga total no membro inferior acometido e abordagem reabilitacional intensiva e precoce. A dor é o principal sintoma 
que interfere no ganho funcional destes doentes. Os três mecanismos básicos responsáveis pela gênese da dor nos doentes em tratamento com o fixador externo tipo Ilizarov são por nocicepção, por desaferentação ou ambos. O acometimento direto ou indireto de estruturas nervosas pelo uso do fixador é causa de dor por desaferentação caracterizada por choque, queimor, pontada ou latejamento em áreas com alterações da sensibilidade.

O objetivo do presente trabalho é o de apresentar os achados clínicos e o resultado terapêutico do ensaio clínico em doentes portadores de dor por desaferentação durante o uso de fixadores externos do tipo Ilizarov, atendidos no Ambulatório de Pseudoartroses e na Divisão de Medicina Física do Instituto de Ortopedia e Traumatologia do Hospital das Clínicas da Faculdade de Medicina da Universidade de São Paulo.

TABELA 1 - TIPO DE DOR

\begin{tabular}{lr}
\hline - Queimor: & 15 \\
- Choque: & 14 \\
- Latejante: & 8 \\
- Pontada: & 3 \\
- Peso: & 3 \\
\hline
\end{tabular}

\section{Casuística e Método}

Vinte e um doentes portadores de dor por desaferentação foram aleatoriamente incluídos no estudo. Foram excluídos os casos de dor apenas por nocicepção. As idades variaram entre 11-58 anos $(M=31,1 \%)$. Havia onze doentes do sexo masculino e nove do sexo feminino. A dor teve início de 2 a 150 dias $(M=43,66 \%)$ após a instalação do fixador externo. A dor por desaferentação era caracterizada por queimor, choque, latejamento, pontada e peso (Tabela 1) acompanhada por alterações da sensibilidade como alodínea, hipoestesia, hiperestesia e hiperpatia (Tabela 2). Os

TABELA 2 DISTÚRBIOS SENSITIVOS

\begin{tabular}{lr}
\hline - Hipoestesia: & 14 \\
- Hiperpatia: & 13 \\
- Hiperestesia: & 4 \\
- Alodínea: & 3
\end{tabular}

territórios nervosos acometidos eram o fibular em 14 casos, obturador em 4, femoral em 2 e safeno em 1 (Tabela 3).

O tratamento consistiu na utilização de antidepressivos tricíclicos associados a neurolépticos fenotiazínicos e antiinflamatórios não hormonais em 14 doentes. Sete receberam apenas

\section{TABELA 3 TERRITÓRIO NERVOSO ACOMETIDO}

- Nervo Fibular:

14

- Nervo Obturador:

- Nervo Femoral:

- Nervo Safeno:

2

1

antiinflamatórios não hormonais. Medidas analgésicas de medicina física como a eletroestimulação do sistema nervoso periférico através de geradores de corrente elétrica de baixa freqüência foram empregados. Compressas de gelo em regime domiciliar por 20 minutos, 3 a 4 vezes por dia e termoterapia profunda com ultrasom foram utilizadas em alguns doentes (Tabela 4). Um doente apresentou quadro de distrofia simpáticoreflexa no membro inferior acometido e recebeu 2 bloqueios simpáticos com guanetidina endovenosa, através da técnica descrita por Hannington Kiff( ${ }^{(2)}$.

Os procedimentos reabilitacionais visando ganho funcional, carga progressiva e marcha eram reiniciados assim que possível.

TABELA 4 - TRATAMENTO

\begin{tabular}{lr}
\hline - Medicamentoso & \\
- ADT + NEUROLÉPTICOS + AINH & 14 \\
- AINH & 7 \\
- Medidas de Medicina Física & \\
- ELETROESTIMULAÇÃO DO SNP & 9 \\
- COMPRESSAS DE GELO & 8 \\
- ULTRASOM & 5 \\
- BLOQUEIO SIMPÁTICO & 1 \\
\hline
\end{tabular}

ADT, antidepressivos tricíclicos; AINH, antiinflamatório não hormonal; SNP, sistema nervoso periférico.

\section{Resultados}

Os resultados foram quantificados segundo a informação dos doentes de acordo com a porcentagem de melhora sintomática da dor. Resultados bons representados por alívio superior a $75 \%$ da dor original foram observados em 16 doentes (76,2\%), de $45-75 \%$ de melhora em 1 doente ( $4,76 \%$ dos casos) e inferior a $45 \%$ em 4 casos (19,04\%). Em 71,4\% dos casos, ocorreu alívio completo da dor. Em nenhum doente houve piora na intensidade da dor (Figura 1).

\section{Discussão}

O conceito de fixação externa data de meados do século XIX com os trabalhos de Malgaigne (1840). Graças ao avanço tecnológico, a fixação 


\section{FIGURA 1 \\ RESULTADOS}

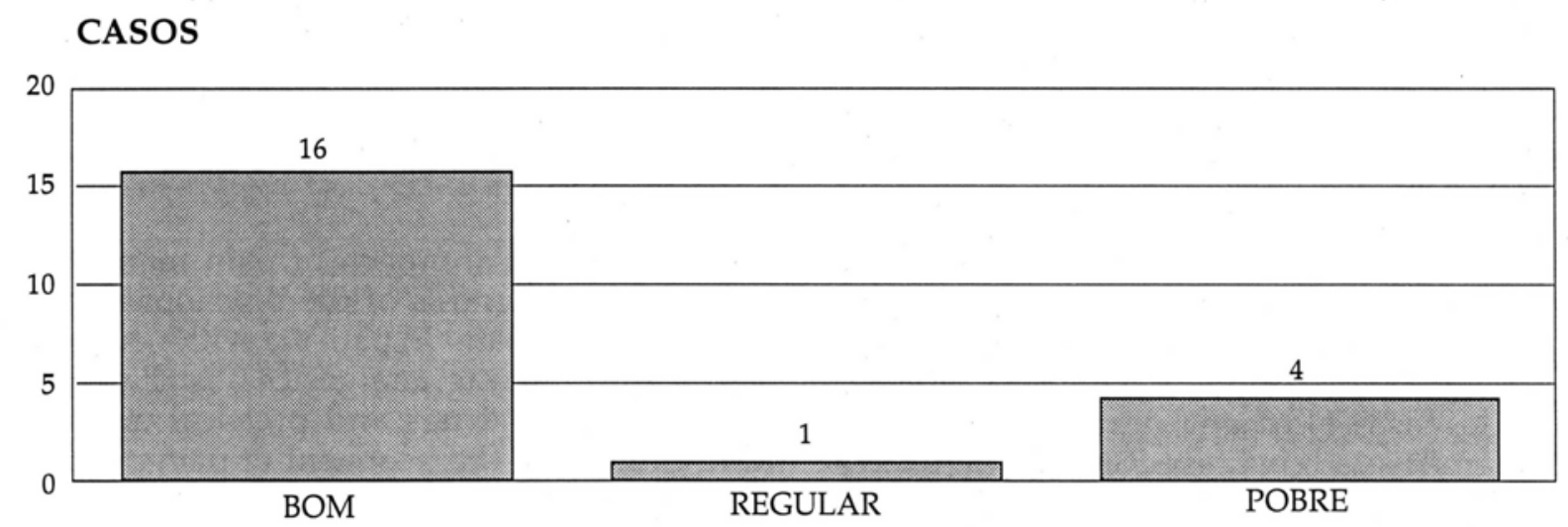

$\mathrm{N}=\mathbf{2 1}$

externa constitui hoje um valioso procedimento terapêutico em diversas patologias do sistema músculo-esquelético. As principais indicações ortopédicas incluem os alongamentos ósseos, nos doentes portadores de acondroplasia e para equalização de dismetrias; deformidades congênitas e adquiridas dos membros inferiores e superiores; deformidades articulares, como nos desvios angulares; contraturas articulares; perda de substância óssea e seqüelas de processos infecciosos ósseos. Na traumatologia, as principais indicações incluem as fraturas, inclusive do anel pélvico e as pseudoartroses, pela possibilidade de compressão e distração do foco de fratura.

Dentre as vantagens da fixação externa em relação às demais formas de tratamento, destacamos a possibilidade de imobilização estável em fraturas infectadas ou com grande desvitalização de tecidos moles; permitindo o controle sistêmico da área afetada, que não fica encoberta por aparelho gessado, além de possibilitar enxertia de pele.

Nos casos de fraturas cominutivas graves e/ ou perda de substância óssea nos quais a fixação interna é desaconselhável ou tecnicamente difícil, a fixação externa é muito útil. Outras vantagens dizem, ainda, respeito à possibilidade de deambulação precoce e mobilização imediata das articulações adjacentes ao foco da fratura, impedindo a instalação de rigidez articular, amiotrofias e osteoporose secundárias à síndrome de imobilização. O fixador externo tipo Ilizarov permite a carga total sobre o membro inferior acometido logo após a sua montagem, já no primeiro dia pós-operatório, garantindo a recuperação funcional precoce e a independência funcional na vigência do uso do fixador.

A marcha $\mathrm{e}$ a atividade nos membros inferiores promovem a ossificação e auxiliam na prevenção de deformidades. A carga auxilia no alongamento dos músculos da panturrilha, mantém o tônus muscular e estimula a circulação nos membros inferiores.

A dor representa a principal causa de retardo da aquisição funcional nos doentes portadores de fixadores externos, principalmente o Ilizarov. Do ponto de vista fisiopatológico, a dor nestes doentes pode ser por aumento de estímulos nociceptivos, por interrupção das vias discriminativas aferentes sensitivas dolorosas (dor por desaferentação) ou pela associação de ambos os mecanismos.

A dor por aumento de nocicepção pode ser decorrente de infecção superficial nos orifícios de entrada e saída dos fios, infiltração local com ou sem infecção, transfixação de estruturas de partes moles, como músculos e tendões. Geralmente o quadro álgico é desencadeado ou exacerbado pela mobilização articular daquele segmento. A dor por desaferentação decorre principalmente da lesão direta de ramúsculos nervosos sensitivos por alguns dos fios ou eventualmente por lesão da "vasa nervorum" secundária a um fenômeno de distração abrupto, principalmente durante alongamentos ósseos. O diagnóstico de dor por desaferentação é feito por meio da avaliação da queixa de dor de características neuropáticas como choque, queimor, pontada ou latejamento, associada a distúrbios da sensibilidade no território nervoso acometido. As principais alterações encontradas na nossa casuística foram a hiperpatia e a hipoestesia. O nervo fibular foi o mais acometido (Tabela 3).

A adequada abordagem terapêutica dependerá do mecanismo fisiopatológico envolvido. Nos casos de dor por desaferentação indica-se medicamentos analgésicos de ação central, como os 
antidepressivos tricíclicos associados aos neurolépticos fenotiazínicos, anti-inflamatórios não hormonais, associados a medidas de neuroestimulação do sistema nervoso periférico e outras medidas de medicina física. $\mathrm{Na}$ nossa casuística, observamos melhora significante, com alívio completo da dor em 15 doentes $(71,4 \%)$. Dois doentes com resultado pobre e um com o resultado regular fizeram uso apenas de medicação antiinflamatória não hormonal pois os mesmos não podiam ou não quizeram fazer uso da medicação analgésica de ação central. O reposicionamento dos fios ou a retirada do fixador não foi necessária em nenhum dos casos. No doente portador de distrofia simpático-reflexa advinda da fixação externa, foi necessário o bloqueio simpático com a guanetidina que é um medicamento simpatolítico.

\section{Conclusão}

Baseando-se nestas observações, os autores concluem ser importante o diagnóstico fisiopatológico dos mecanismos envolvidos na gênese da dor. Nos doentes portadores de dor por desaferentação o uso de medicação analgésica de ação central associada aos antiinflamatórios não hormonais e medidas de medicina física foram eficazes no tratamento sintomático da dor, o que permitiu a permanência do fixador externo e a continuidade do tratamento ortopédico.

\section{Conclusion}

The rational approach of pain management includes the diagnosis of the physiopathological mechanisms of pain. In patients with neurophatic pain, central acting analgesics associated with antiinflamatory drugs and physical modalities were effective in the treatment of pain permitting the use of the external fixator.

\section{Referências Bibliográficas}

1. GOLYAKLOVSKY, V; FRANKEL, V.H.: Fixator removal and complication of llizarov technique. In Operative Manual of llizarov techniques, Ed. Mosby, New York, chapter 10, 1993.

2. HANNINGTON-KIFF, G.: Intravenous regional block with guanethidine, Lancet 1, p. 1019-1020, 1974.

3. PALEY, D.: Problems, obstacles and complications of limb lengthening by llizarov technique. Clin Orth 250, p. 81-104, 1990. 